\title{
OXYBUOY: CONSTRUCTING A REAL-TIME INEXPENSIVE HYPOXIA MONITORING PLATFORM
}

\author{
A thesis submitted \\ to Kent State University in \\ partial fulfillment of the requirements \\ for the degree of Master of Science
}

by

Rizal Mohd Nor

Dec 2009 
Thesis written by

$$
\text { Rizal Mohd Nor }
$$

B.S., Johns Hopkins University, 2000

M.B.A, International Islamic University Malaysia, 2004

M.S., Kent State University, 2009

Approved by

Mikhail Nesterenko , Advisor

Robert A. Walker , Chair, Department of Computer Science

John Stalvey , Dean, College of Arts and Sciences 


\section{TABLE OF CONTENTS}

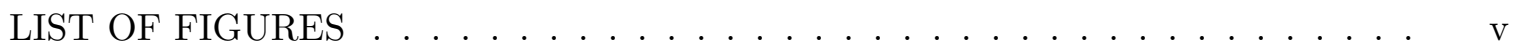

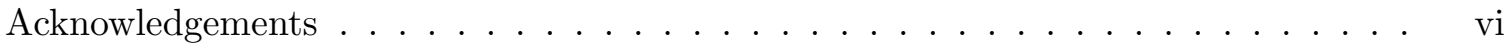

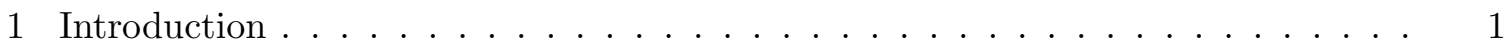

2 Unattended Sensors and Their Applications . . . . . . . . . . . . . . . 4

2.1 Microsensors . . . . . . . . . . . . . . . . . . . 4

2.2 Midsize Sensors . . . . . . . . . . . . . . . . . . . . . . 7

2.3 Marine Application of Sensor Nodes . . . . . . . . . . . . . . . . . . 10

3 Oxybuoy Description . . . . . . . . . . . . . . . . . . . 13

4 Oxybuoy Implementation $\ldots \ldots \ldots \ldots$

4.1 Architecture Description . . . . . . . . . . . . . . . 18

4.2 Electric Power Design . . . . . . . . . . . . . . . . . . . . . . . . 19

4.3 Programming and Operation . . . . . . . . . . . . . . . 20

4.4 Buoy Construction and Sensor Device Container Description . . . . . . . 20

5 Experimental Setup . . . . . . . . . . . . . . . . . . 24

5.1 Controlled DO Lab Experiment . . . . . . . . . . . . . . . . . . 24

5.2 Bath Lake Deployment . . . . . . . . . . . . . . . . . . . 26

5.3 Power Consumption Study . . . . . . . . . . . . . . . . . . . 26

6 Conclusion and Future Work f . . . . . . . . . . . . . . . . . . 29 


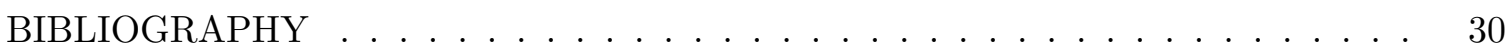




\section{LIST OF FIGURES}

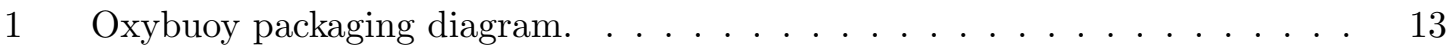

2 Data consolidation diagram. . . . . . . . . . . . . . . . . 14

3 Data visualization on web page using JpGraph. . . . . . . . . . . . . . 15

4 Data center software architecture. . . . . . . . . . . . . . 16

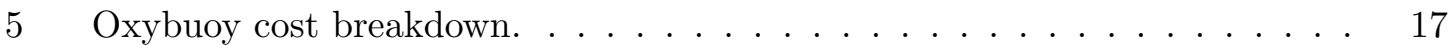

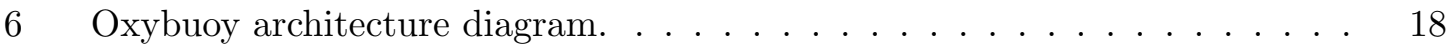

7 Oxybuoy satellite transmission algorithm. . . . . . . . . . . . . 21

$8 \quad$ Lake deployment experiment. . . . . . . . . . . . . . . . . . . 22

9 Packaging of oxybuoy electronics for the DO lab experiment. . . . . . . . 24

10 Oxybuoy measurements in the DO lab experiment. . . . . . . . . 25

11 Oxybuoy measurements in the lake deployment experiment. . . . . . . . . 27

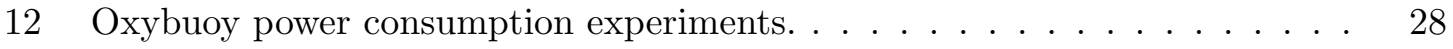




\section{Acknowledgements}

First and foremost I would like to thank my advisor Dr. Mikhail Nesterenko for making this thesis possible by providing initial direction and constant guidance during the course of this work. A special thanks goes to Dr. Peter Lavrentyev from University of Akron for all his help and support in the understanding of Hypoxia studies. A special thanks goes to Dr. Brian Bagatto and Francisco Moore of the University of Akron for allowing us to use their lab and water tanks for the experiments and their other assistance. 
Dedicated to my family for all their support. 


\section{CHAPTER 1}

\section{Introduction}

Hypoxia, i.e. dissolved oxygen (DO) depletion in the lower part of the water column caused by terrestrial, mostly anthropogenic, nutrient loading is an emerging national and global problem [3]. Reduced oxygen availability negatively affects biological resources, including fish [4] and commercially important invertebrate species [5]. To mitigate the environmental impact of hypoxia, the phenomenon requires extensive study. However, the dynamics of hypoxia development is poorly understood. Hypoxia tends to occur in large areas of coastal waters. For example, in the northern Gulf of Mexico, the hypoxic zone can reach $20,000 \mathrm{~km}^{2}$ [6]. Traditional approaches to monitor DO concentrations rely on using research vessels to collect water samples for subsequent chemical analysis or to trawl DO sensors behind the vessel. These approaches necessarily limit the resolution of time-series data as they are expensive due to the vessel operating costs and man power involved. The resolution of the data is limited further due to the measurements being weather-dependent as the ship carrying DO sampling gear cannot be operated in rough seas. Furthermore, it is impossible to measure DO concentration in several diverse locations simultaneously with a single research vessels. The lack of data that can match the scale of the phenomena hinders the study and the efforts to correctly model hypoxia.

Remote sensing is commonly used in large-scale oceanographic studies. For example, Pearce and Pattiaratchi [7] discuss various satellite image processing techniques to determine sea-surface temperature, surface chlorophyll distribution and ocean's currents. However, satellite data require ground truthing and are limited to the ocean surface, whereas hypoxia develops in bottom waters. Unlike sea temperature and phytoplankton photopigments, DO 
does not have a proxy suitable for remote observations. Relationships between satellitemeasured chlorophyll and hypoxia are confounded by the interaction of physical processes with the seasonal cycle of nutrient-enhanced productivity [6]. The limitations of traditional approaches stress the need for new high-resolution real-time monitoring tools.

An unattended buoy system becomes an attractive option. An array of such buoys deployed in coastal areas can record the DO concentration around the clock at multiple locations simultaneously and relay the data to the researcher. The technical requirements for such a buoy are as follows. A buoy needs to be capable of independent operation for up to four months - the time hypoxia occurs. It needs to be positioned 10 to 40 miles offshore and capable of sensing DO at depths of up to 50 meters. Four to six times per day sampling rate is acceptable. All these parameters are well within what present-day technology allows. However, the market for such buoys is so small that the commercial companies have to charge large premiums to recoup their development costs. Another commercial strategy is to develop a sensor platform that could be used for a variety of studies. Such platform tends to contain features that are not necessary for hypoxia study. Both approaches push the price of a commercial buoy outside the price range where extensive hypoxia studies become feasible. Meanwhile, advances in consumer electronics and sensor technology enable such buoy construction from commercial off-the-shelf components at costs that make large hypoxia studies practical.

Thesis contribution. In this thesis I describe Oxybuoy: A real-time inexpensive oxygen monitoring platform. Unlike previous autonomous platforms used to acquire sensor data in remote areas, Oxybuoy provides a inexpensive solution to implement a unattended sensor and is capable of functioning many miles off shore for an extended period of time. Oxybuoy is constructed using commercial off-the-shelve (COTS) components and can be assembled without extensive electronic expertise. The total cost of the buoy is around $\$ 5,000$. Most of 
this cost, $\$ 3,500$ was due to the DO sensor itself. Communication equipment, the satellite modem and the antenna cost $\$ 750$, the rest of the electronics was a bit under $\$ 650$ and the casing was around $\$ 100$.

We illustrate the operation of Oxybuoy with three experiments as proof of concept. The first experiment is a laboratory tank deployment where the data collected was compared to the data in the tank where the environment was controlled by a pre-installed control system. The second experiment is a lake deployment to test the transmission of collected data over satellite and the third experiment is done in a laboratory to test the power consumption of the device.

The rest of this thesis is organized as follows. In Chapter 2, we discuss previous advances in sensor network research and their applications. In Chapter 3, we describe the Oxybuoy system and its operation. Oxybuoy design considerations are discussed in Chapter 4. In Chapter 5, we describe our experiments with the buoy. In the last chapter, Chapter 6, we outline our future research plans. 


\title{
CHAPTER 2
}

\author{
Unattended Sensors and Their Applications
}

The unattended sensors can be divided into several classes based on their size and capabilities. Microsensors are small scale devices with low power requirements, processing, communication and storage capacity. Midsize sensor nodes have a higher power requirement but they have greater communication, storage and processing capabilities. There is a large number of diverse applications, where both microsensors and midsize sensors can be used successfully. In this chapter, we describe the common architecture and applications for microsensors and for midsize sensors. We then focus on marine based applications.

\subsection{Microsensors}

The advances of processor manufacturing and sensing technology have enabled the construction of microsensors for the use in sensor networks. Researchers today are able to deploy these microsensors devices equipped with sensors and radio transmitters to measure the physical phenomena in the environment, gather data, relay to human operators as well as use actuators to influence this environment [8-10]. Microsensors can be used in a wide range of applications from military surveillance [11-13] to environmental monitoring [14-16].

A single microsensor is limited in terms of processing speed, storage capacity and communication bandwidth. However, this makes their manufacturing cost very low. Due to their low cost, microsensors can be deployed in large numbers: hundreds, thousands or even hundreds of thousands of units. In such large numbers, the intended sensor nodes tend to be located closer to to the observed phenomenon. The collected sensor data provides a far greater resolution than a few large scale sensors are able to provide. These allow the 
microsensor technology to provide solutions to problems that were previously impossible to solve.

The most common application of sensor networks is monitoring [14-20]. Among the most frequently used devices are Berkeley prototype sensor nodes called motes [21,22]. Motes are a convenient research and development platform because they are flexible and easy to operate. There are several families of motes: Mica, Mica2, Mica2Dot and MicaZ. All of them share a common Atmel ATMega128L microprocessor with a $32 \mathrm{kHz}$ crystal and $4 \mathrm{Mhz}$ crystal except for Mica2 which has a higher crystal clock of $7.37 \mathrm{MHz}$. The motes have a 10-bit ADC, two UART channels, an SPI bus and an I2C bus. All of them have a external serial flash memory of 512 KBytes. The motes have 3 programmable LEDs, except Mica2Dot, which has only one LED, to help in debugging. All motes have I/O connectors to interface with sensors and a programming board. Mica has an RFM TR1000 radio transceiver and is capable of radio communication in the frequency range from $902-928 \mathrm{MHz}$ and 433.1 - 434.8 MHz. Mica2 and Mica2Dot provide better communication capabilities with the Chipcon CC1000 radio transceiver. They could operate on frequencies ranges which was from $868-870 \mathrm{MHz}, 902-928 \mathrm{MHz}, 433.1-434.8 \mathrm{MHz}$ and $313.9-316.1 \mathrm{MHz}$. MicaZ provided a better range with a higher frequency in the range of $2400-2483.5 \mathrm{MHz}$ using the Chipcon CC2420 transceiver. MicaZ is also the first in Mica family to support the IEEE 802.15.4 protocol.

All of the motes model run the TinyOS operating system [23] which was specifically designed for such resource constrained devices. TinyOS is designed to be power efficient, modular, has a small memory footprint and is capable of supporting concurrency-intensive operations. Although TinyOS does not provide any hard real-time guarantees, it does support real-time query and feedback control of physical world. An application in TinyOS is a combination of the scheduler and a graph of components compiled into one executable. The architecture is event-driven with a single shared stack. TinyOS does not have kernel 
and user space differentiation.

Motes have frequently been used on land for monitoring purposes. One of the first successful deployment of motes is the habitat monitoring application developed by Culler et al. [15]. This application recorded the temperature and humidity in a nature preserve. Mica motes were deployed on the Great Duck Island, located $15 \mathrm{~km}$ South of the Mount Desert Island, Maine. Mica motes were equipped with photoresistors, temperature sensors, barometric pressure sensors, humidity sensors and thermistors. The island is a protected area which is restricted to human presence. Therefore, the use of unattended microsensors was desirable. The use of these sensors allowed researchers to gather long term data at a greater scale and resolution. This micro-scale data enables biologist to improve and control the habitat. Culler et al. [24] later extended their deployment with higher number of nodes to study the use of microsensors on a large scale.

Culler et al. [20] used Mica2Dot to monitor micro-climate in Redwood coastal canopy. The microsensors were deployed throughout a 70 meter high redwood tree to monitor temperature, light and humidity. Results were later gathered to perform analysis of spatial and temporal trends. The analysis provides valuable information for studying the micro-climatic conditions in the coast of redwood trees.

Chazko and Ahmad [25] used Mica2 motes to create a sensor network to get an early warning system for fire endangered areas. The use of micro-sensors deployed in fire endangered areas can provide real-time data for bush-fire fighting. They indicated that microsensor networks can be use in hostile environments.

Culler et al. [26] showed that microsensors can also be used for infrastructure health monitoring. The sensors were equipped with two accelerometers to measure vibrations and detect movements in two axis on the bridge. A total of 59 nodes were deployed. One of the challenges is filtering out the noise. Temperature sensor were used to calibrate the accelerometers for operation at different temperatures. To achieve desirable quality of 
data, extensive analog and digital signal processing techniques were implemented. Another challenge is to transmit the data with minimum jitter: the required sampling rate was higher than the transmitting rate, hence the device have to alternate between sampling and transmitting.

Priya and Inman [27] from Energy Harvesting Technologies described promising use of energy harvesting sensor network devices. They designed, developed and demonstrated the use of a structural health monitoring system for military helicopters. The devices are designed for long term operation, without the need of a battery. They harvest energy from vibrations using micro fiber composite material. The devices have strain sensors to measure strain on the structure and a 2 MByte flash memory to save the compressed data. The data is transmitted to the nearest gateway when available using WiFi transceiver via IEEE 802.15.4 protocol. The device is able to handle high sampling rates and capable of operating in energy saving mode when there is insufficient energy to be harvested.

Estrin et al. [28] described the use of Mica2 modes for a soil sensing system. The researchers provided a systematic design description of a robust sensing system suited for the challenges of harsh agricultural environment. The researchers described 3 separate long term deployment of sensors at a rice paddy field in Bangladesh, James Reserve and San Joanquin River basin in California. They presented techniques to maximizing sensor yield by improving calibration techniques and dealing with calibration drift. They have managed to increase the yield of the network of sensors to achieve a delivery ratio of $91 \%$ by implementing a delay-tolerant network and monitoring data flow from one node to another.

\subsection{Midsize Sensors}

The emergence of low power processors for embedded computers like Gumstix [29] and Stargate [30] enabled a wide variety of applications from PDAs and cellphones to sensors, actuators and robotics. These devices are less resource constrained. They usually have 
greater processing power, larger memory storage, and better communication range than microsensors. However, these devices has a higher power requirements. Midsize sensors may require a large battery source or a solar panel.

The advantage of using these midsize sensors is their capability to interface other devices and to ease programming. Embedded computers such like the Gumstix and Stargate has several serial communication ports, a general purpose input output (GPIO) bus, an I2C bus, ADC channels and IEEE 802.11 communication capabilities. These extra features allow them to be easily integrated with other devices such as sensor, actuator or a GPS. The embedded computer usually support full-fledged operating system such as Linux operating system or the Windows CE. Unlike regular PCs, midsize sensors are robust and can operate in harsh conditions.

For example, the Gumstix board is capable of operating at low temperature of $-25^{\circ} \mathrm{Cel}-$ sius to a high temperature of $85^{\circ}$ Celsius. Williams, Viguria and Howard [31] relied on Gumstix embedded chip for operating a robotic mobile sensor network to take measurements in the arctic. The weather data from glacial regions can provide important and valuable information on global climate change. Acquiring these data is challenging because of the harsh environment and inaccessible terrain. Traditional methods of human expeditions to collect data have been short duration, limited in area, slow and dangerous. Another traditional methods such as fixed weather stations provides continuous data feed. However, it has low spatial resolution. Hence a robotic mobile sensor network was desirable to provide greater resolution on specific regions of the arctics. The Gumstix board were mounted on a RC snowmobile. The Gumstix board can interface with weather sensor units, GPS control and camera feedback to navigate the harsh terrain of the arctic. For low level control on navigational motors, I2C communication is used with microcontrollers. Mobile sensor nodes communicates with one another using the IEEE 802.11 WiFi communication protocol.

Midsize sensors are small enough to be used as wearable computers. Fraser et al. [32] 
utilized the Gumstix board to create a wearable device to track in $2 \mathrm{D}$ and $3 \mathrm{D}$, the locations of a marker pen. The sensor were carefully disguised as a marker pen and helped in keeping track of a presenters' drawing on a screen. The marker pen was equipped with a Gumstix board, battery, bluetooth aerial, and a ultrasonic receiver to help in $3 \mathrm{D}$ positioning and tracking of the marker pen. To create a system capable of tracking the pen around the display, 6 transmitters were used to send ultrasonic pulses which are received by the Gumstix embedded computer. The Gumstix calculates the time differential to determine the receiver's position with respect to the known transmitter locations. The researchers were able to demonstrate improvements in response time, accuracy, and predictability of their tracking application.

Gumstix is designed with flexibility in mind. This makes Gumstix an attractive platform for sensor applications. Hughes et al. [33] used the Gumstix board in creating a flood monitoring network where external sensors are attached to a Gumstix processor to detect dangerous water levels. Flood is a growing problem which causes lost of billions of dollars by affecting land use and damaging properties. The damages incurred correlates with the warning time, depth and the flow speed of the flood. Current methods to detect and measure water levels are sparse and does not cover sufficient flood areas. Therefore, there is a need to develop a flood monitoring sensor network to improve measurements of areas with high risk for flood and improve flood prediction models to mitigate lost. There were 4 types of sensors used on the flood monitoring sensor. The pressure base depth sensor with lighting strike protection and the conductivity-based pollution sensor connects via the Analog to Digital Converter(ADC) channels on the Gumstix board. The ultrasound-based flow monitoring sensor interfaced the Gumstix board via RS232. The digital camera for image-based flow measurement are connected to the Gumstix board via Ethernet port. The sensor nodes were powered by a $12 \mathrm{~V} 7 \mathrm{Ah}$ gel battery and a $12 \mathrm{~V}$ solar array. The researchers demonstrated benefits on having such a sensor network in reconfiguring flood models. 


\subsection{Marine Application of Sensor Nodes}

Challenges of marine sensor design. Marine based sensors pose a new set of challenges for sensor network deployment. Electronics can easily become damaged when coming in contact with water. Thus, the packaging in marine based sensors is particularly important. The packaging should withstand constant dynamic movement of the sensor as well as insulate the device from moisture. Wires from the electronics connecting to external sensors should be carefully designed to assure safety of equipment from water penetrating into the casing.

The second challenge is providing power. Supplying wired power to a marine based sensor is seldom feasible. Replacing batteries is also cumbersome as it requires visiting the sensor at the place of deployment. This is either expensive or otherwise undesirable. Using solar panels is a viable solution but it increases the sensor cost.

Communication is a major challenge for marine based sensors. The communication means for marine sensors are limited. If the device is underwater, one of the most common way to communicate with it is by using an acoustic modem [34]. Wills, Ye, and Heidemann [35] described extensive use of acoustic modems between marine sensors. However, acoustic modems have a number of short comings: their range is limited, their susceptible to noise and their latency is high. Hence their usage is only feasible for short range communication.

Another option is to use IEEE 802.11 wireless communication protocol. For this the antenna needs to be installed above the water surface. However, the antenna needs to be placed high enough so that the Fresnel zone is unaffected by the water surface. Fresnel zone is an area around the line-of-sight between two RF antennas within which obstructions degrade the strength of the signal. The surface of the water itself is such an obstruction. The radius of the Fresnel Zone is largest at the midpoint between the antennas and smallest at 
the end-points at the two antennas. The radius of the Fresnel Zone at the midpoint between the two antennas is given by formula 1

$$
h=72.1 \times \sqrt{\frac{L}{4 F}}
$$

where $h$ is the radius of the Fresnel zone in feet, $L$ is the distance between the antennas in miles, and $F$ is the frequency of the RF signal in GHz. A common guideline is that the Fresnel zone must be at least $60 \%$ clear of obstructions, so the 72.1 multiplier in the above equation is commonly substituted with 43.3 to give the $60 \%$ radius of the Fresnel zone. Hence, the further apart the antenna are, the higher they need to be placed. This requires the construction of high mast which makes the deployment expensive. For example, for two antennas 1 mile apart transmitting at $2.437 \mathrm{GHz}$ using IEEE $802.11 \mathrm{~b}$ communication protocol, the Fresnel radius at the middle of the 1 mile link would be about 13.9 feet. Therefore, the antennas should be placed at least 13.9 feet high. Another option for communication for marine sensors is satellite modems. However, satellite communication can be expensive if there is a lot of data to be transmitted.

Marine sensor deployments. There is a number of midsize and microsensor marine deployment reported in literature. Bokser et al. [36] used Mica2 to developed a small submarine robot for the experiments in underwater sensor networks. Besides Mica2, the submarine robot had a pressure sensor, a thermistor and a propulsion piston to change its' buoyancy. The robot was capable of maintaining desired depth level in the water by sensing the pressure on the pressure sensor and maintaining its' depth by activating the pistons to react to any change in pressure. The experiment demonstrated the viability of using microsensors for underwater sensor networks.

Graber et al. [37] described a compact buoy called the ASIS capable of measuring atmospheric data in the open ocean. Gobat et al. [38] reported on the development of an 
extensive military meteorological buoy systems. Guinasso et al. [39] and Bender et al. [40] described a series of large buoy deployments for surface current measurements. Wallinga et al. [41] described a buoy platform that can accommodate up to 100 sensors. This is a largesize buoy which requires solar batteries to power the equipment. Blain et al. [42] described the design of a DO sensor MAREL buoy. The design was done using a membrane-based DO sensor. Such sensors are sensitive to bio-fouling and long-term measurement drift. The buoy contains a system of bio-fouling prevention that involves pumping the water from the desired depth to the surface for measurement, then automatic periodic flushing of the sensor with an anti-bacterial chlorinated solution. There is also the need for extensive sensor calibration to account for measurement drift. The data was transmitted from the MAREL buoy to shore using wireless LAN technology. Voigt et al. [43] described their experiences building an array of temperature-measuring buoys connected by a wireless LAN.

In their position paper, Akyildiz et al. [44] described the challenges of gathering sensor data in the marine environment. Wood et all [45] described a design of a self-propelled float or a canoe that can follow a certain route and en route lower the sensor with a winch to a desired depth and take measurements. Another automated sensor gathering system is described by Vasilescu et al [46]. It involves robotic submarine, optical and acoustic communication, etc. These designs may be too expensive and complicated for the kind of hypoxia studies we anticipate. 


\section{CHAPTER 3}

\section{Oxybuoy Description}

The Oxybuoy is designed to operate autonomously to measure oxygen concentration in a body of water for extended duration. Illustrated in Figure 1, the Oxybuoy is equipped with COTS components to measure oxygen concentration, measure water temperature, record and manage data collected, manage power usage, facilitate satellite communication transmission, and interaction with data server for data consolidation.

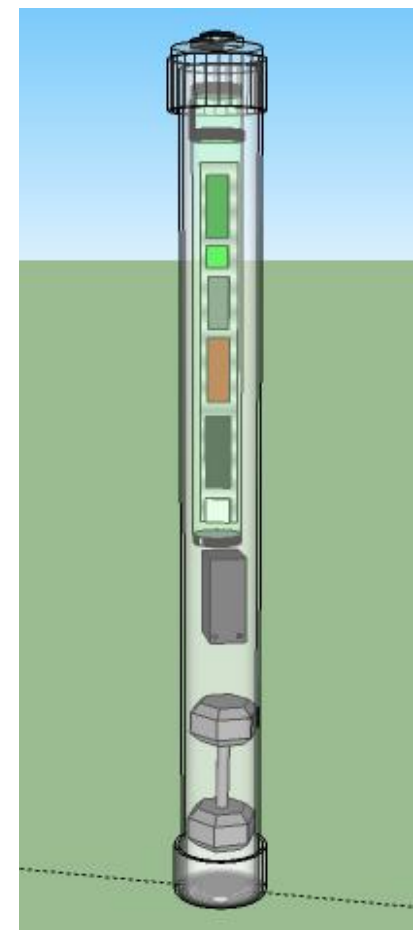

Figure 1: Oxybuoy packaging diagram.

Oxybuoy can be deployed far away from the shore as it uses a satellite modem to transfer the data to a data center anywhere in the world. Figure 2 illustrates the consolidation of data across several Oxybuoys deployed at sea. Consolidated data can be used to visualize 
the effect of hypoxia in higher resolution and extended area coverage.

The data center can also send certain commands to reprogram the deployed Oxybuoys. Messages can be sent to all deployed Oxybuoys or to individual buoy. These messages convey commands to change sampling times in case it is necessary to record the DO level at a different rate, for example when a rapid change of oxygen concentration is detected.

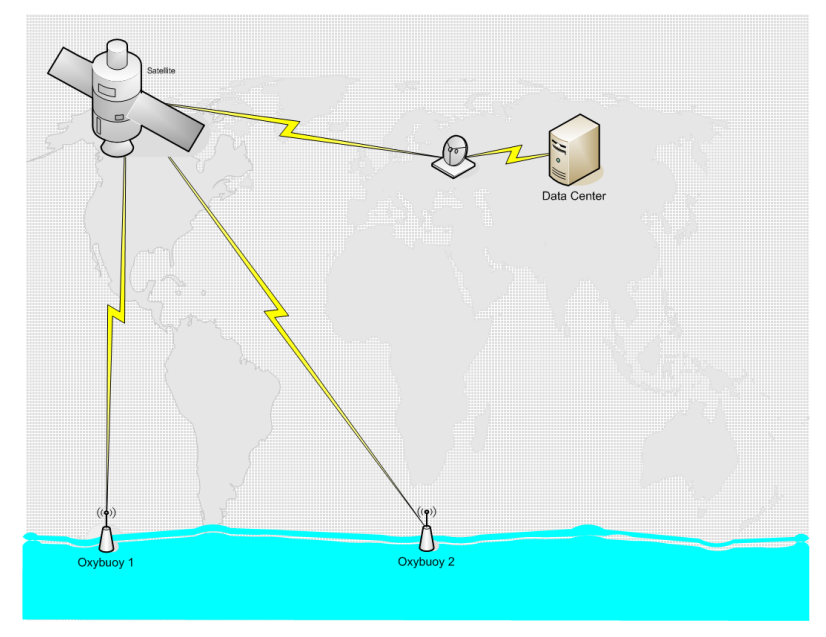

Figure 2: Data consolidation diagram.

The data is sent to the data center where a server helps in extracting raw data and visualizes the data to a web browser. The server has a Linux operating system hosting an Apache web server [47], a MySQL database [48] server and a PHP [49] script interpreter. The combination of these technologies, Linux, Apache, MySQL and PHP, is more commonly known as LAMP. Using the LAMP web architecture, I wrote PHP scripts on the host, to query the database for relevant information. I used a graphing utility library called JpGraph [50] to plot and present the data in a graph at real-time. The graph is then displayed on a web browser. The illustration of the whole architecture is shown in Figure 4. The output of the graph is displayed in a web browser as shown in Figure 3.

Design decisions. Our design decisions were dictated by the hypoxia study requirements 


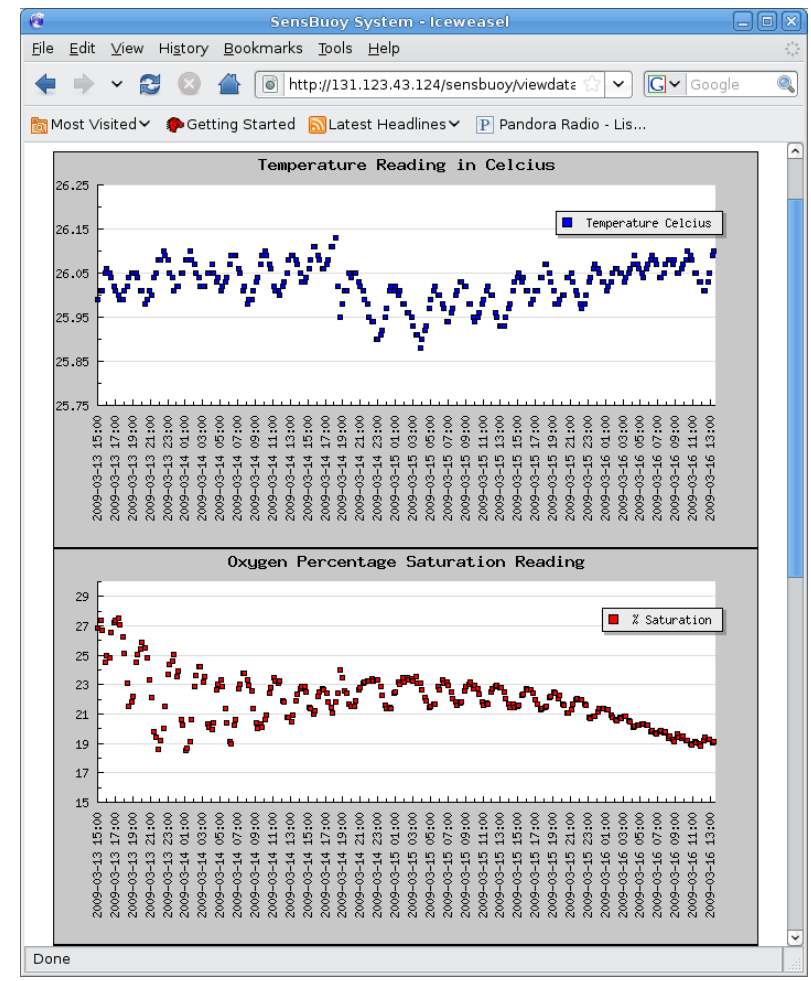

Figure 3: Data visualization on web page using JpGraph.

and the need to keep the buoy cost as low as possible. We used an optical dissolved oxygen sensor. This type of DO sensor, although relatively expensive, has fewer problems with bio-fouling and loss of calibration than the commonly used membrane-based DO sensors do. There is a relatively limited amount of data to be collected during DO monitoring. Coupled with low sampling rate, it allowed us to use simple sealed batteries and avoid having to install solar panels.

Let us discuss our buoy communication decisions. Oxybuoy needs to be deployed in such distances from the shore that cellular networks are not reachable. Wireless communication at such distances requires high masts and other design options that would make the cost of the buoy prohibitively expensive. However, due to the limited data rate, we are able to use commercial satellite network for data transmission.

Oxybuoy components can be purchased from well known vendors at high volumes, 


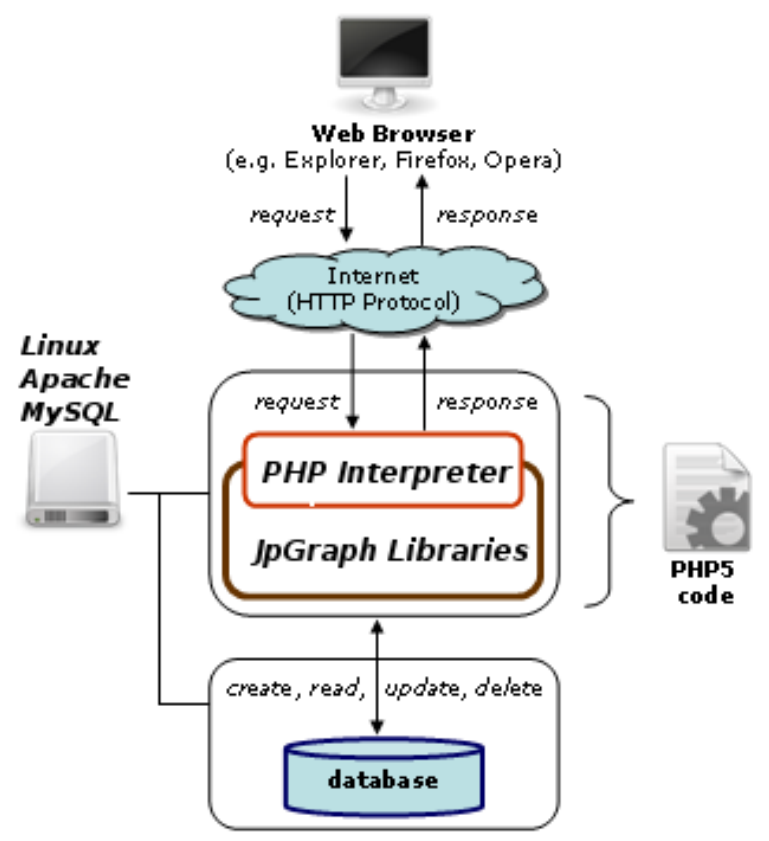

Figure 4: Data center software architecture.

thereby making the construction of Oxybuoy even cheaper. For the prototype, the cost of each component is listed in Figure 5. 


\begin{tabular}{|l|l|c|c|r|}
\hline Hardware & Description & Units & \multicolumn{1}{c|}{ Price } & \multicolumn{1}{c|}{ Total } \\
\hline Device Casing & Watertight casing & 1 & $\$ 85.90$ & $\$ 85.90$ \\
\cline { 2 - 5 } & Watertight water seal & 3 & $\$ 15.00$ & $\$ 45.00$ \\
\hline Electronics Devices & Gumstix embedded board & 1 & $\$ 350.00$ & $\$ 350.00$ \\
\cline { 2 - 5 } & SDI to RS232 converter & 1 & $\$ 99.95$ & $\$ 99.95$ \\
\cline { 2 - 5 } & PIC microcontroller 16F86 & 1 & $\$ 10.00$ & $\$ 10.00$ \\
\cline { 2 - 5 } & $\begin{array}{l}\text { 12 volts 32 Ah gel } \\
\text { Gel cell sealed lead } \\
\text { Acid battery }\end{array}$ & 2 & $\$ 73.95$ & $\$ 147.90$ \\
\cline { 2 - 5 } & $\begin{array}{l}\text { 5V 1A switching } \\
\text { voltage regulator }\end{array}$ & 2 & $\$ 15.00$ & $\$ 30.00$ \\
\hline Communication Devices & 9601DN satellite modem & 1 & $\$ 500.00$ & $\$ 500.00$ \\
\cline { 2 - 5 } & SAF5350 antenna & 1 & $\$ 250.00$ & $\$ 250.00$ \\
\hline Sensor Devices & D-Opto sensor & 1 & $\$ 3500.00$ & $\$ 3500.00$ \\
\hline \multicolumn{2}{|l|}{ Grand Total } & $\$ 5018.75$ \\
\hline
\end{tabular}

Figure 5: Oxybuoy cost breakdown. 


\section{CHAPTER 4}

Oxybuoy Implementation

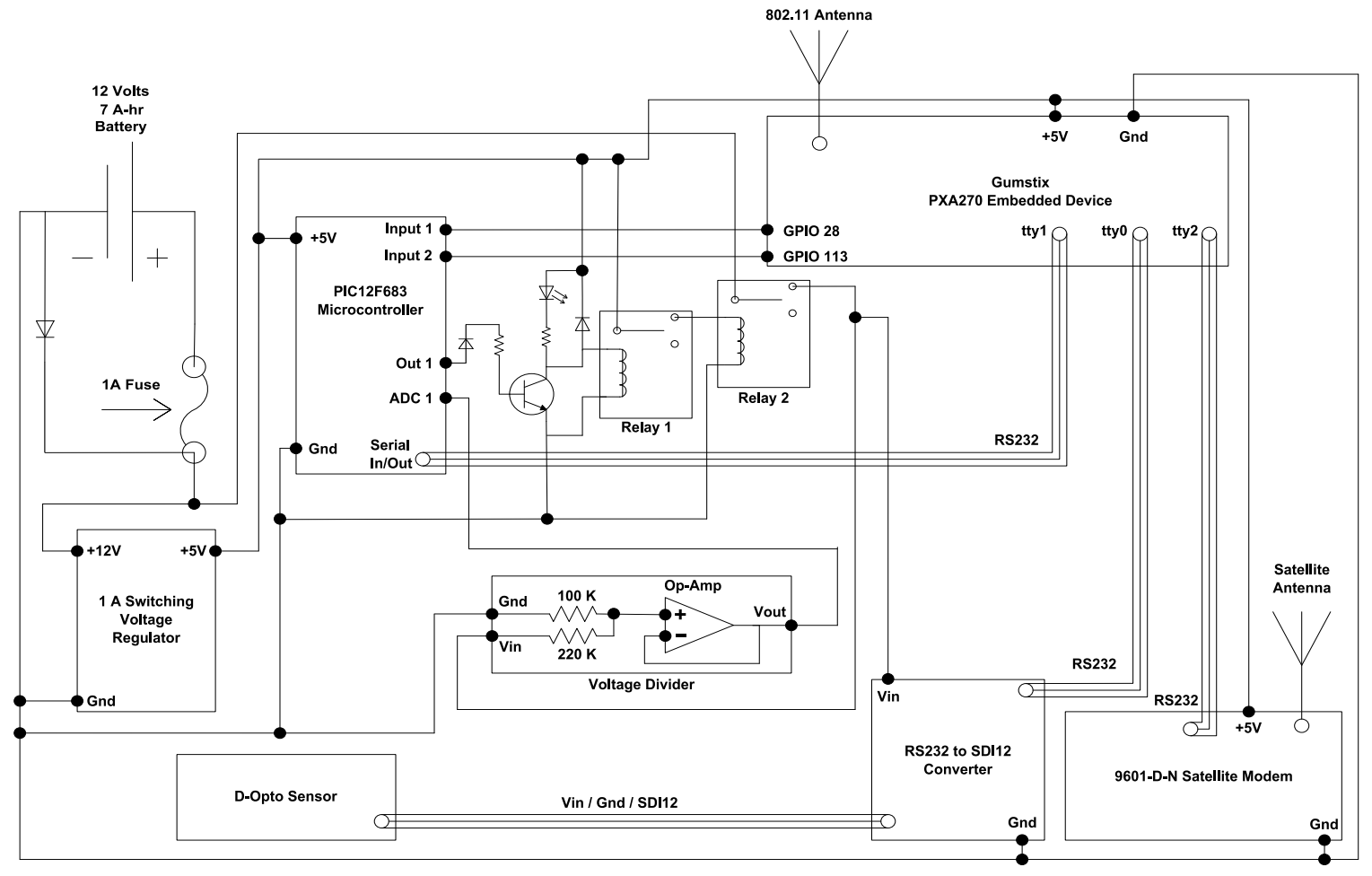

Figure 6: Oxybuoy architecture diagram.

\subsection{Architecture Description}

The Oxybuoy electronics design contains the following main components: Gumstix Marvell XScale PXA270 processor embedded system [29], PIC12F683 Microcontroller [51], 9601D-N Satellite Modem [52], RS232 to SDI Converter, Marvell 88W8385 802.11(b and g) wireless card, D-OPTO optical sensor [53], switching relay and a switching voltage regulator. Oxybuoy architecture diagram is shown in Figure 6.

The main data processing and control over the other devices is carried out by the 
Gumstix embedded system. Two Gigabyte SD flash card connected to the Gumstix provide data storage for the system.

The optical sensor is controlled via SDI-12 sensor communication standard. To connect the sensor to the Gumstix we use RS232 to SDI-12 converter. For real-time transmission of data, a satellite modem is used. The satellite modem is connected directly to one of Gumstix serial ports. The satellite modem allows real-time data to be transmitted to a server for data consolidation. The wireless card allows backup and debugging communication channel to the system. The PIC processor is used to implement the low-power operation of the system. Gumstix and PIC processors complement each other. Gumstix provides flexible user and programmer interface and ease of data storage and retrieval while PIC has low power consumption.

\subsection{Electric Power Design}

There are two modes of the system operation: active sampling mode and power saving sleep mode. The system alternate between these tho modes. In the active sampling mode the system draws about $350 \mathrm{~mA}$, while in the sleep mode the system draws only $11 \mathrm{~mA}$. The current drawn is measured using an ammeter connected in series with the device. During the sleep mode, the PIC microprocessor is the only component that remains powered on. The PIC microcontroller keeps track of the clock cycle for the next sampling time. The PIC has 1024-bit ADC connection to the battery. It is capable of reading the voltage level there. This information is helpful in determining the remaining charge left in the battery. The PIC is programmed to switch the system to the active sampling mode. It then sends the current battery voltage level to the Gumstix processor and waits for a 2-bit signal from it to indicate the sleep period. After receiving this signal, the PIC switches the system to sleep mode by powering down the remainder of the system.

Most of the system components require reliable 5V DC power. The DO sensor requires 
$8 \mathrm{~V}$ power. We use a $5 \mathrm{~V} 1 \mathrm{~A}$ switching voltage regulator to provide stable voltage to the system. This regulator has conversion efficiency of up to $90 \%$ which helps to minimize power consumption.

\subsection{Programming and Operation}

Gumstix main processor runs an Open Embedded Linux operating system, a flavor of Linux for embedded systems, which allows us to easily interface programming environments on the Linux kernel. Programs and scripts are written and loaded into Open Embedded to handle communication between devices and system maintenance tasks. The system operation is as follows. When Gumstix is powered up, it receives the ADC battery power level data from the PIC processor. Then Gumstix communicates through the RS232 serial connector to the SDI-12 converter to request a reading from the DO sensor. After collecting the data, Gumstix requests the satellite modem to transmit the data to the base station. During satellite communication, the Gumstix checks the signal strength indicator.

If it is too low, the data is saved on the SD card and transmitted during the next communication session. After satellite communication, the Gumstix issues the command to the PIC informing it of the sampling rate and instructing it to power down the system.

During its uptime Gumstix powers up the wireless card and remains accessible over the wireless channel for status checks and configuration updates. Gumstix is programmed to receive control commands from the satellite. In particular the sampling rate can be changed remotely. The algorithm for the satellite transmission is shown in Figure 7.

\subsection{Buoy Construction and Sensor Device Container Description}

To protect the electronics from water exposure we had to design the buoy packaging. The buoy packaging is shown in Figure 8. The buoy casing is constructed using a 6" PVC pipe that can be purchased from any hardware store to work as an outer shell.

For the electronics container we used a simple poster carrier pipe shown in Figure 8(a). 


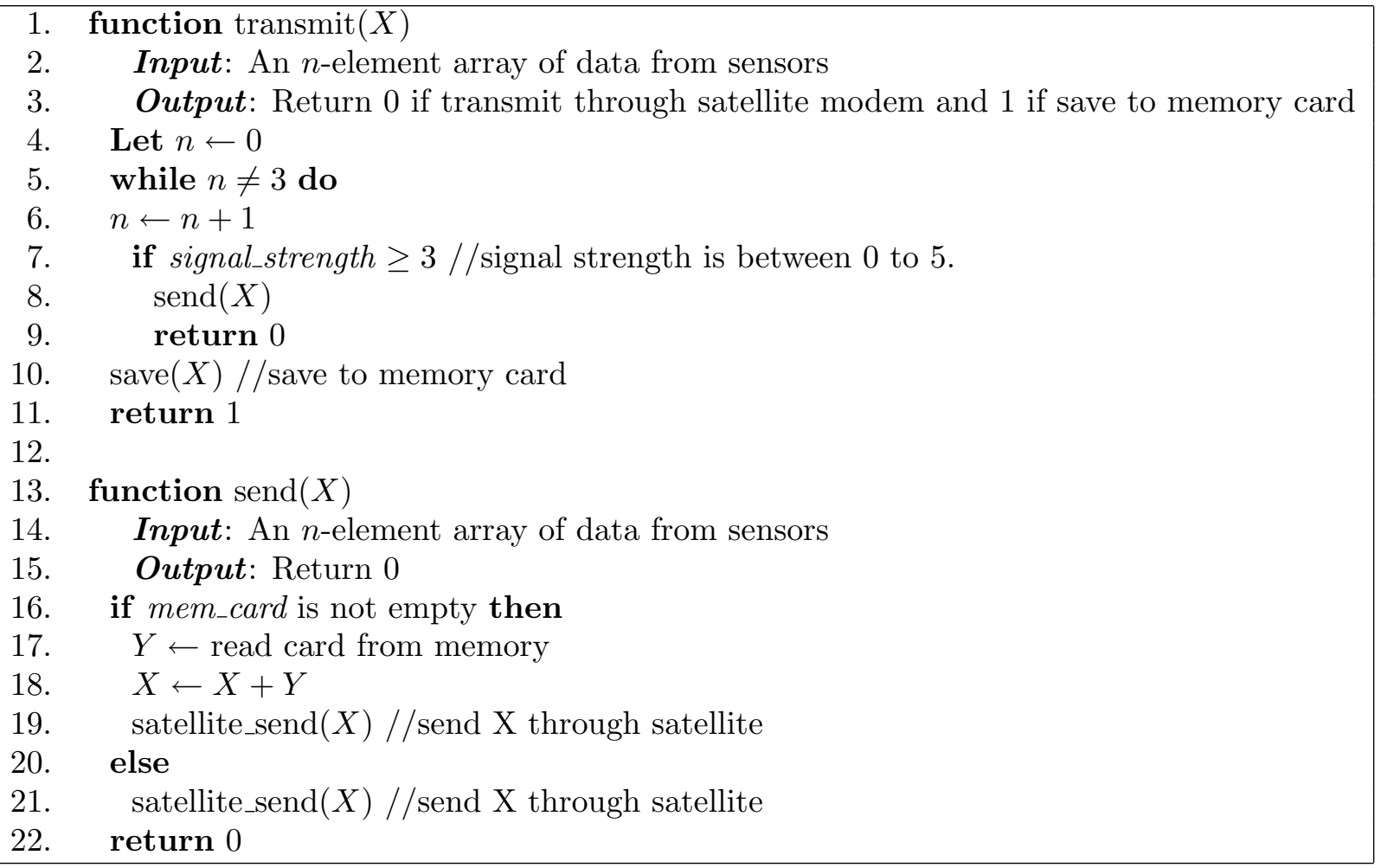

Figure 7: Oxybuoy satellite transmission algorithm. 


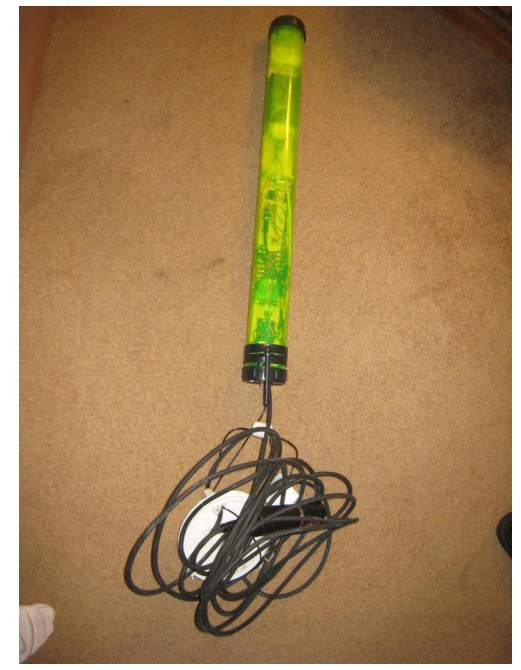

(a) Electronics packaging of Oxy- (b) Oxybuoy ready for assembly
buoy.

Figure 8: Lake deployment experiment.

The electronics container is later fitted into the buoy. Styrofoam forms are used to keep the electronics from moving inside the casing.

The outer pipe housed the electronics, ballast and the battery. The buoy was attached by a chain to a single anchor point. The outer shell was hermetically sealed at the bottom. The oxygen sensor, and the satellite antenna cables were fed through the silicone-sealed hole in the screw-on pipe cover. This way all the possible orifices are above water.

PVC pipe adhesives are used to glue together the pipe bottom end to make the internals of the buoy water proof. We also had to ensure that the buoy is submerged deep enough in the water so that it is stable yet the buoy cover with the instrument and antenna openings are high enough above the water level to prevent continuous contact with it. The correct weight of a the ballast for the buoy would have to be carefully calculated.

We computed the weight of the ballast to have the buoy submerged about three-fourths in the water. The calculation is based on the amount of water the buoy displaces, and hence the amount of buoyancy it provides. We measured the volume of the pipes, and estimated the amount of water that would be displaced. We measured the weight of the buoy along 
with the weight of the equipment to be installed in the buoy. Lastly, we tested the ballast using weights. Sandbags were used as weights necessary to fine tune the amount of required ballast. After getting the correct measurement for the ballast, we replaced the sandbags with the exact amount of weight. 


\section{CHAPTER 5}

\section{Experimental Setup}

This section describes the series of experiments that we carried out to determine the viability and performance of Oxybuoy. In the first experiment we used a controlled laboratory environment to check the operation of the Oxybuoy's electronics. In the second we performed a test deployment in a small lake. In the third experiment we ran an extended power usage measurements. The first experiment was wall powered while the last 2 experiments relied on the $12 \mathrm{~V}$ DC, 7 Ah sealed battery.

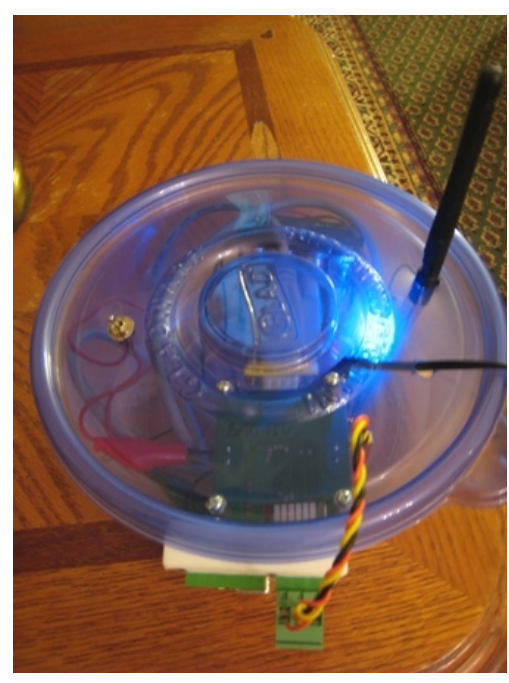

Figure 9: Packaging of oxybuoy electronics for the DO lab experiment.

\subsection{Controlled DO Lab Experiment}

The objective of this experiment was to test the operation of the electronics of Oxybuoy in the controlled environment. We used a water tank in a fish physiology laboratory at the University of Akron equipped for hypoxia experiments. The DO concentration in the tank was maintained at a specific level. The tank had external thermometer and YSI [54] DO 
meter. Before the experiment we calibrated our DO sensor according to the manufacturer instructions.

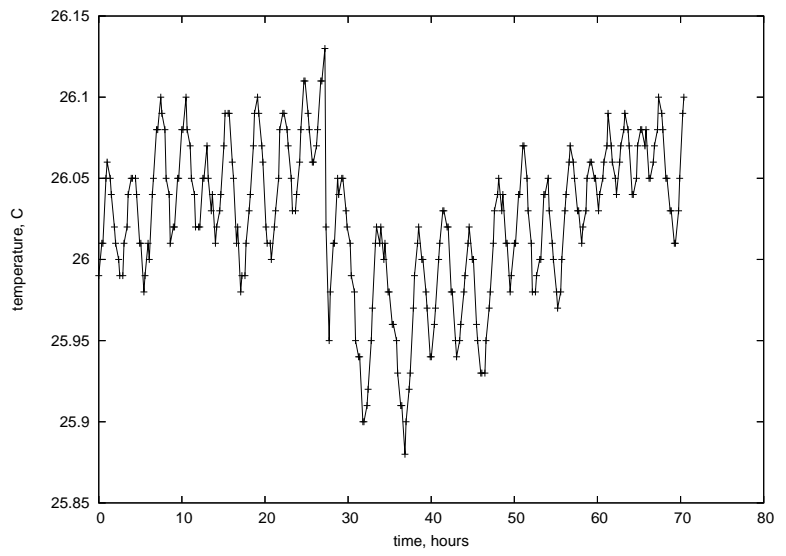

(a) Temperature readings.

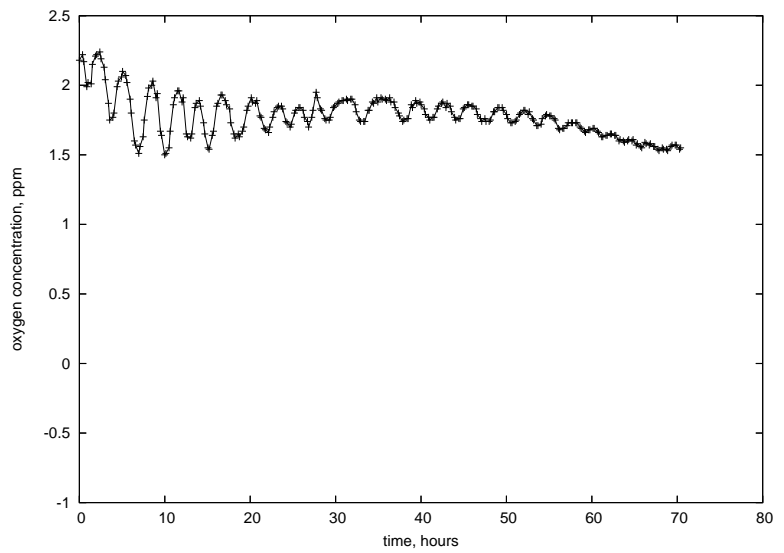

(b) DO concentration readings.

Figure 10: Oxybuoy measurements in the DO lab experiment.

Minimal protective packaging was used for the electronic components (see Figure 9). Only the DO sensor itself was submerged. We configured Oxybuoy to use the wireless card to report the measurements every 20 minutes to the wireless bridge and on to the Data Center where the information was collected. The collected DO and temperature data are shown in Figure 10.

The oscillation of temperature and DO concentration level are due to the design of the tank system: periodically a motor pumps fresh water into the tank. The temperature 
readings agree with the external meter measurements precisely while the DO concentration measurements agree with them approximately.

\subsection{Bath Lake Deployment}

The objective of this experiment was to test the complete operation of Oxybuoy in the target environment. We deployed the buoy in Bath Lake, a small eutrophuc lake within the Bath Nature Preserve near Akron, Ohio for 7 days. After the week-long deployment the design proved generally sound. Although, some moisture permeated the outer shell, the electronics was not damaged. We first tested the seal of the outer shell in a separate short deployment. Before the main deployment we tested the operation of the electronic components through first wireless connection then through the satellite messages.

During the deployment we did not use the multi-mode operation due to faulty communication port on our Gumstix board. We used active sampling mode only and allowed the buoy to run until the battery charge was exhausted. During the deployment, Oxybuoy reported DO measurements 6 times per hour. Running in this mode, Oxybuoy remained operational and reported DO measurements for over 18 hours. The measurements are shown in Figure 11. For testing purposes Oxybuoy started transmitting the measurements before it was deployed. Thus, the early reported data differs from the rest. The reported tempera-

ture values agree with the expectations. The DO concentration was different from expected, which may have been due to the DO sensor lense periodically touching the sediment.

\subsection{Power Consumption Study}

To estimate the lifetime of the buoy in multi-mode operation we ran the electronics of the buoy in the simulated deployment. The electronics were configured to switch to data acquisition mode once an hour. The DO sensor was submerged into a small water container. The PIC processor recorded the battery power output and relayed it to the Gumstix processor. The Gumstix transmitted this value over the wireless network. We 


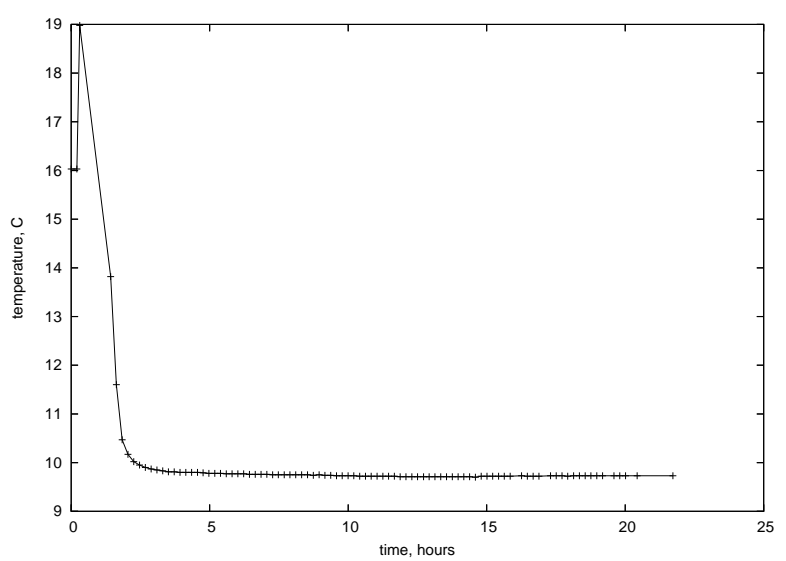

(a) Temperature readings.

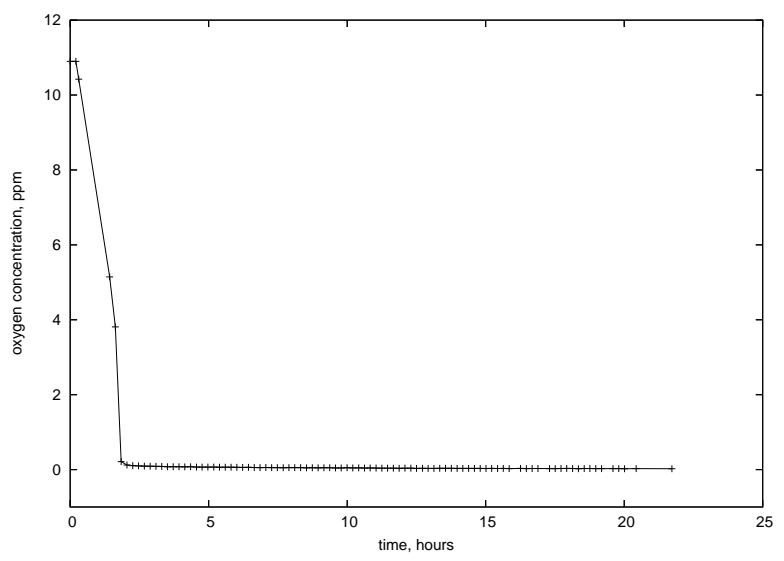

(b) DO concentration readings.

Figure 11: Oxybuoy measurements in the lake deployment experiment.

stopped the experiment when the battery power output fell below 8 Volts required by the DO sensor to operate. The results are shown in Figure 12. Oxybuoy produced the total of 155 samples. At the rate of sample per hour, Oxybuoy can operate over six days.

This result agrees with the calculations. The battery is rated at $7 \mathrm{Ah}$. For this amount of time the battery is able to provide at least $10.5 \mathrm{~V}$. During the active sampling mode the average current draw of Oxybuoy is $350 \mathrm{~mA}$. It takes about 6 minutes to sample the DO sensor and transmit the data. This allows 120 samples. We are able to obtain more samples since we are able to use the battery until the voltage fell below $8 \mathrm{~V}$. On the basis of the above data we can predict the long term-operation of the buoy. If the buoy samples every 


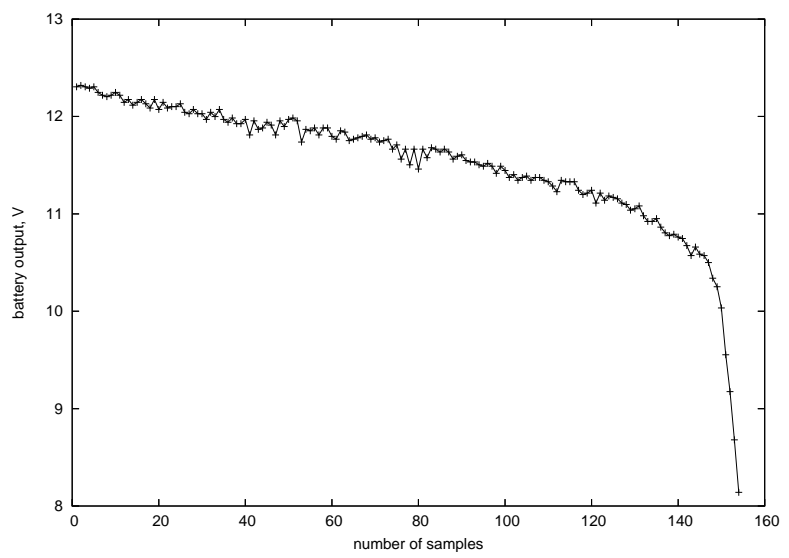

Figure 12: Oxybuoy power consumption experiments.

hour, its 4 month operation requires a 160 Ah battery. If only four times a day sampling is required, 28 Ah battery would be sufficient. 


\section{CHAPTER 6}

\section{Conclusion and Future Work}

In this thesis we described the architecture and deployment history of a prototype buoy for dissolved oxygen sensing. The experiments demonstrate the viability of this sensor

platform but further studies are required. In the future we plan to upgrade our sensor platform so that it is capable of unattended DO measurements for the complete duration of annual hypoxia occurrence. We plan to deploy the new buoy in both freshwater and marine environments and use it in hypoxia measurements of practical importance. 


\section{BIBLIOGRAPHY}

[1] R. Mohd Nor, M. Nesterenko, and P. Lavrentyev, "Oxybuoy: Constructing a real-time inexpensive hypoxia monitoring platform, technical report, computer science department, kent state university," Kent State University, Kent, Ohio, USA, Tech. Rep. TR-KSU-CS-2009-01, 2009. [Online]. Available: http://www.cs.kent.edu/ techreps/TR-KSU-CS-2009-01.pdf

[2] R. Mohd Nor, M. Nesterenko, and P. Lavrentyev, "Oxybuoy: Constructing a real-time inexpensive hypoxia monitoring platform," in ADHOCNETS 2009, ser. Lecture Notes of the Institute for Computer Sciences, Social-Informatics and Telecommunications Engineering (LNICST). Springer, 2009.

[3] R. J. Diaz and R. Rosenberg, "Spreading dead zones and consequences for marine ecosystems," Science, vol. 321, pp. 926-929, 2008.

[4] P. Thomas, S. Rahman, I. A. Khan, and J. A. Kummer, "Widespread endocrine disruption and reproductive impairment in an estuarine fish population exposed to seasonal hypoxia," Proceedings of the Royal Biological Society, vol. 274, pp. 2693-2702, 2007.

[5] T. OConnor and D. Whitall, "Linking hypoxia to shrimp catch in the northern Gulf of Mexico," Marine Pollution Bulletin, vol. 54, pp. 460-463, 2007.

[6] N. Walker and N. Rabalais, "Relationships among satellite chlorophyll a, river inputs and hypoxia on the louisiana continental shelf, gulf of mexico," Estuaries and Coasts, vol. 29, pp. 1081-1093, 2006.

[7] A. Pearce and C. Pattiaratchi, "Applications of satellite remote sensing to the marine enviornment in western australia," Journal of the Roayl Society of Western Australia, vol. 80, pp. 1-14, 1997.

[8] D. E. Culler, D. Estrin, and M. B. Srivastava, "Guest editors' introduction: Overview of sensor networks." IEEE Computer, vol. 37, no. 8, pp. 41-49, 2004.

[9] D. Estrin, R. Govindan, J. S. Heidemann, and S. Kumar, "Next century challenges: Scalable coordination in sensor networks," in MOBICOM, 1999, pp. 263-270. [Online]. Available: http://doi.acm.org/10.1145/313451.313556

[10] D. E. Culler and W. Hong, "Wireless sensor networks - introduction," Commun. ACM, vol. 47, no. 6, pp. 30-33, 2004. [Online]. Available: http://doi.acm.org/10.1145/990703

[11] A. Arora, P. Dutta, S. Bapat, V. Kulathumani, H. Zhang, V. Naik, V. Mittal, H. Cao, M. Gouda, Y. Choi, T. Herman, S. Kulkarni, U. Arumugam, M. Nesterenko, A. Vora, and M. Miyashita, "A line in the sand: a wireless sensor networking for target detection, classification, and tracking," Computer Networks, Special Issue on Future Advances in Military Communication and Technology, vol. 46, no. 5, pp. 605-634, December 2004. 
[12] J. Chhabra, N. Kushalnagar, B. Metzler, and A. Sampson, "Sensor networks in intel fabrication plants," in SenSys, J. A. Stankovic, A. Arora, and R. Govindan, Eds. ACM, 2004, p. 324. [Online]. Available: http://doi.acm.org/10.1145/1031495.1031567

[13] M. Maroti, G. Simon, Á. Lédeczi, and J. Sztipanovits, "Shooter localization in urban terrain." IEEE Computer, vol. 37, no. 8, pp. 60-61, 2004.

[14] R. C. Oliver, K. Smettem, M. Kranz, and K. MAyer, "A reactive soil moisture sensor network: Design and field evaluation," International Journal of Distributed Sensor Networks, vol. 1, pp. 149-162, Apr./Jun. 2005.

[15] A. Mainwaring, D. Culler, J. Polastre, R. Szewczyk, and J. Anderson, "Wireless sensor networks for habitat monitoring," in Proceedings of the First ACM International Workshop on Wireless Sensor Networks and Applications (WSNA-02). New York: ACM Press, Sep. 28 2002, pp. 88-97.

[16] S. M. Brennan, A. M. Mielke, D. C. Torney, and A. B. Maccabe, "Radiation detection with distributed sensor networks." IEEE Computer, vol. 37, no. 8, pp. 57-59, 2004.

[17] A. Arora, R. Ramnath, P. Sinha, E. Ertin, S. Bapat, V. Naik, V. Kulathumani, H. Zhang, M. Sridharan, S. Kumar, H. Cao, N. Seddon, C. Anderson, T. Herman, C. Zhang, N. Trivedi, M. Gouda, Y.-R. Choi, M. Nesterenko, R. Shah, S. Kulkarni, M. Aramugam, L. Wang, D. Culler, P. Dutta, C. Sharp, G. Tolle, M. Grimmer, B. Ferriera, and K. Parker, "Project exscal (short abstract)," in Proceedings of Distributed Computing in Sensor Systems, First IEEE International Conference, (DCOSS), ser. Lecture Notes in Computer Science, vol. 3560. Marina del Rey, CA, USA: Springer, June 2005, pp. 393-394.

[18] M. Batalin, M. Rahimi, Y. Yu, D. Liu, A. Kansal, G. Sukhatme, W. Kaiser, M. Hansen, G. Pottie, M. Srivastava, and D. Estrin, "Call and response: Experiments in sampling the environment," in Proceedings of the 2nd International Conference on Embedded Networked Sensor Systems, (SenSys). Baltimore, MD, USA: ACM, November 2004, pp. 25-38.

[19] T. He, S. Krishnamurthy, J. Stankovic, T. Abdelzaher, L. Luo, R. Stoleru, T. Yan, L. Gu, J. Hui, and B. Krogh, "Energy-efficient surveillance system using wireless sensor networks," in Proceedings of the International Convference on Mobile Systems, Appications and Services (MobiSys). USENIX, June 2004.

[20] G. Tolle, J. Polastre, R. Szewczyk, D. Culler, N. Turner, K. Tu, S. Burgess, T. Dawson, P. Buonadonna, D. Gay, and W. Hong, "A macroscope in the redwoods," in Proceedings of the 3rd International Conference on Embedded Networked Sensor Systems, (SenSys). Sand Diego, California, USA: ACM, November 2005, pp. 51-63.

[21] J. Hill and D. Culler, "Mica: A wireless platform for deeply embedded networks," IEEE Micro, vol. 22, no. 6, pp. 12-24, Nov./Dec. 2002.

[22] http://www . xbow . com/Products/productdetails . aspx?sid=174.

[23] J. Hill, R. Szewczyk, A. Woo, D. Culler, S. Hollar, and K. Pister, "System architecture directions for networked sensors," ACM SIGPLAN Notices, vol. 35, no. 11, pp. 93-104, Nov. 2000. 
[24] R. Szewczyk, A. Mainwaring, J. Polastre, J. Anderson, and D. Culler, "An analysis of a large scale habitat monitoring application," in SenSys '04: Proceedings of the 2nd international conference on Embedded networked sensor systems. New York, NY, USA: ACM, 2004, pp. 214-226.

[25] Z. Chaczko and F. Ahmad, "Wireless sensor network based system for fire endangered areas," Information Technology and Applications, International Conference on, vol. 2, pp. 203-207, 2005.

[26] S. Kim, S. Pakzad, D. Culler, J. Demmel, G. Fenves, S. Glaser, and M. Turon, "Health monitoring of civil infrastructures using wireless sensor networks," in IPSN '07: Proceedings of the 6th international conference on Information processing in sensor networks. New York, NY, USA: ACM, 2007, pp. 254-263.

[27] S. Priya and D. J. Inman, "Energy harvesting wireless sensors," in Energy Harvesting Technologies. Springer US, 2009, pp. 195-208.

[28] N. Ramanathan, D. E. T. Schoellhammer, T. H. M. Hansen, and M. S. E. Kohler, "The final frontier: Embedding networked sensors in the soil," UCLA, Tech. Rep., November 2006. [Online]. Available: 22CAFC26D4A093DAE0406180528D790D

[29] Gumstix Inc, "Gumstix verdex pro xm4-bt (with u.fl antenna)," http://www.gumstix. com/store/catalog/product_info.php?products_id $=206$.

[30] Crossbow Technology Inc, https://www.xbow.com/.

[31] A. V. Stephen Williams and A. M. Howard, "A robotic mobile sensor network for achieving scientific measurements in challenging environment," in Proc. of the Earth Science Technology Conference (ESTC 2008), 2008.

[32] M. Fraser, M. R. McCarthy, M. Shaukat, and P. Smith, "Seconds matter: improving distributed coordination bytracking and visualizing display trajectories," in $C H I$ ' $0 \%$ : Proceedings of the SIGCHI conference on Human factors in computing systems. New York, NY, USA: ACM, 2007, pp. 1303-1312.

[33] D. Hughes, P. Greenwood, G. Coulson, and G. Blair, "Gridstix: Supporting flood prediction using embedded hardware and next generation grid middleware," in the proceedings of the 4th International Workshop on Mobile Distributed Computing (MDC'06), Niagara Falls, 2006.

[34] J. Heidemann, W. Ye, J. Wills, A. Syed, and Y. Li, "Research challenges and applications for underwater sensor networking," in Proceedings of the IEEE Wireless Communications and Networking Conference. Las Vegas, Nevada, USA: IEEE, April 2006, pp. 228-235. [Online]. Available: http://www.isi.edu/ johnh/PAPERS/ Heidemann06a.html

[35] J. Wills, W. Ye, and J. Heidemann, "Low-power acoustic modem for dense underwater sensor networks," in WUWNet '06: Proceedings of the 1st ACM international workshop on Underwater networks. New York, NY, USA: ACM, 2006, pp. 79-85. 
[36] G. S. V. Bokser, C. Oberg and A. Requicha, "A small submarine robot for experiments in underwater sensor networks," in Symposium on Intelligent Autonomous Vehicles, July 2004. [Online]. Available: 005

[37] H. C. Graber, E. A. Terray, M. A. Donelan, W. M. Drennan, and J. C. Van Leer, "Asis-a new air-sea interaction spar buoy: Design and performance at sea." Journal of Atmospheric \& Oceanic Technology, vol. 17, no. 5, pp. 708 -, 2000. [Online]. Available: http://search.ebscohost.com/login.aspx?direct=true $\& \mathrm{db}=$ eih\&AN $=5648732 \&$ si\% te $=$ ehost-live

[38] J. I. Gobat, R. A. Weller, B. S. Way, J. Lord, M. Pritchard, and J. Smith, "A compact coastal ocean observing system for kernel blitz," 2001. [Online]. Available: http://hdl.handle.net/1912/36

[39] J. Guinasso, N.L., J. Yip, R. Reid, I. Bender, L.C., M. Howard, I. Lee, L.L., J. Walpert, D. Brooks, R. Hetland, and R. Martin, "Observing and forecasting coastal currents: Texas automated buoysystem (TABS)," in OCEANS, MTS/IEEE Conference and Exhibition, vol. 2. IEEE, 2001, pp. $1318-1322$.

[40] L. C. B. III, J. Norman L. Guinasso, J. N. Walpert, L. L. L. III, R. D. Martin, R. D. Hetland, S. K. Baum, and M. K. Howard, "Development, operation, and results from the texas automated buoy system," Gulf of Mexico Science, vol. 1, pp. 33-60, 2007.

[41] J. P. Wallinga, N. R. Pettirew, and J. D. Irish, "The GoMOOS moored buoy design," in $O C E A N S$, vol. 5, 2003, pp. 2596-2599.

[42] S. Blain, J. Guillou, P. Trguer, P. Woerther, L. Delauney, E. Follenfant, O. Gontier, M. Hamon, B. Leilde, A. Masson, C. Tartu, and R. Vuillemin, "High frequency monitoring of the coastal marine environment using the MAREL buoy." J Environ Monit, vol. 6, no. 6, pp. 569-75, 2004.

[43] T. Voigt, F. Österlind, N. Finne, N. Tsiftes, Z. He, J. Eriksson, A. Dunkels, U. Bamstedt, J. H. Schiller, and K. Hjort, "Sensor networking in aquatic environments - experiences and new challenges," in Second IEEE International Workshop on Practical Issues in Building Sensor Network Applications. IEEE Computer Society, 2007, pp. 793-798.

[44] I. F. Akyildiz, D. Pompili, and T. Melodia, "Underwater acoustic sensor networks: research challenges," Ad Hoc Networks, vol. 3, no. 3, pp. 257-279, 2005.

[45] S. Wood and Z. P. Michelle Rees, "An autonomous self-mooring vehicle for littoral and coastal observations," in OCEANS, Europe, June 2007, pp. 1-6.

[46] I. Vasilescu, K. Kotay, D. Rus, M. Dunbabin, and P. Corke, "Data collection, storage, and retrieval with an underwater sensor network," in Proceedings of the 3rd international conference on Embedded networked sensor systems (SenSys). New York, NY, USA: ACM, 2005, pp. 154-165.

[47] The Apache Software Foundation, https://www.apache.org/.

[48] Sun Microsystem Inc., https://www.mysql.com/. 
[49] The PHP Group, https://www.php.net/.

[50] Aditus Consulting, https://www.aditus.nu/jpgraph/index.php.

[51] Microchip Technology Inc, http://www.rev-ed.co.uk/picaxe/.

[52] Nal Research Corporation, "9601-d-n satellite sbd modem," http://nalresearch.com/ IridiumHardware.html.

[53] Zebra-Tech LTD., http://www.d-opto.com/.

[54] YSI Inc, https://www.ysi.com/. 